\title{
New Soft-Core Potential Function for Molecular Dynamics Based Alchemical Free Energy Calculations
}

\author{
Vytautas Gapsys, $^{\dagger}$ Daniel Seeliger, ${ }^{\dagger \dagger}$ and Bert L. de Groot ${ }^{*}{ }^{\dagger}$ \\ ${ }^{\dagger}$ Computational Biomolecular Dynamics Group, Max Planck Institute for Biophysical Chemistry, Göttingen, Germany \\ ${ }^{\ddagger}$ Department of Lead Identification and Optimization Support, Boehringer Ingelheim Pharma GmbH, Birkendorfer Strasse 65, 88397 \\ Biberach, Germany
}

Supporting Information

ABSTRACT: The fields of rational drug design and protein engineering benefit from accurate free energy calculations based on molecular dynamics simulations. A thermodynamic integration scheme is often used to calculate changes in the free energy of a system by integrating the change of the system's Hamiltonian with respect to a coupling parameter. These methods exploit nonphysical pathways over thermodynamic cycles involving particle introduction and annihilation. Such alchemical transitions require the modification of the classical nonbonded potential energy terms by applying soft-core potential functions to avoid singularity points. In this work, we propose a novel formulation for a soft-core potential to be applied in nonequilibrium free energy calculations that alleviates singularities, numerical instabilities, and additional minima in the potential energy for all combinations of nonbonded interactions at all intermediate alchemical states. The method was validated by application to (a) the free energy calculations of a closed thermodynamic cycle, (b) the mutation influence on protein thermostability, (c) calculations of small ligand solvation free energies, and (d) the estimation of binding free energies of trypsin inhibitors. The results show that the novel soft-core function provides a robust and accurate general purpose solution to alchemical free energy calculations.

\section{INTRODUCTION}

The estimation of free energies is an important aspect of modern computational chemistry. Alchemical methods based on Molecular Dynamics (MD) or Monte Carlo sampling allow accurate calculations of free energies. ${ }^{1-4}$ The alchemical methods are often applied in predictions of protein-ligand binding free energies, ${ }^{5-8}$ which are of great importance in rational drug design. ${ }^{2,9-11} \mathrm{MD}$-based alchemical methods are also used to estimate free energies of ligand solvation, ${ }^{12-15}$ the influence of mutations on protein thermostability, ${ }^{16}$ or ion permeation through channels. ${ }^{17}$

MD-based computational methods for free energy calculations can be divided into equilibrium and nonequilibrium approaches. Free energy perturbation (FEP) (also called exponential averaging ${ }^{3}$ ) introduced in 1954 by Zwanzig $^{18}$ is an equilibrium method allowing the estimation of the free energy difference between two discrete states. The method suffers from insufficient sampling when the phase space overlap is low, that is, when the perturbation is large. ${ }^{19,20}$ In case of an insufficient overlap between the ensembles, the Hamiltonian $(H)$ of a system can be coupled to a parameter $\lambda$, which allows introduction of intermediates along the path between the two end states of the system. This enables calculation of the free energy between the end states as a sum of the free energy differences between the intermediate states. Another approach is to use a minimum variance estimate, Bennett's acceptance ratio (BAR), ${ }^{21}$ which was shown to be more efficient than the perturbation method. ${ }^{22}$

Another branch of equilibrium approaches is based on thermodynamic integration (TI). ${ }^{23}$ TI methods also couple the Hamiltonian of the system to a parameter $\lambda$ and integrate over the derivative of Hamiltonian with respect to the coupling parameter along the transition path. In case the path between the states $\mathrm{A}$ and $\mathrm{B}$ is traversed by changing $\lambda$ value from 0 to 1 continuously by small steps in $\lambda$, the system stays in a quasiequilibrium state (slow-groth TI). Another way is to select discrete $\lambda$ values along the path between the states and perform simulations at those states only (discrete TI).

The branch of nonequilibrium methods for free energy calculations takes its roots from the equations derived by Jarzynski. $^{24,25}$ A more general formulation was provided by Crooks $^{26}$ and is known as the Crooks Fluctuation Theorem (CFT). The CFT relates the free energy difference with the forward and reverse work distributions for the nonequilibrium transitions between the states $\mathrm{A}$ and $\mathrm{B}$ :

$$
\frac{P_{\mathrm{f}}(W)}{P_{\mathrm{r}}(-W)}=\mathrm{e}^{\beta(W-\Delta F)}
$$

Work values are estimated by integrating change of the Hamiltonian with respect to the coupling parameter $\lambda$ :

$$
W=\int_{0}^{1} \frac{\partial H}{\partial \lambda} d \lambda
$$

The transitions between the states exploit nonphysical pathways, hence the name "alchemical" free energy calculations. When atoms are created or annihilated during an alchemical transition, points of singularity in the $\partial H / \partial \lambda$ may occur if no modifications to the classical nonbonded interactions are made. Also, strong repulsive forces may appear, which would require the integration time step to be modified to avoid numerical

Received: March 16, 2012

Published: May 28, 2012 
instabilities during the simulations. The reason for the singularities is the classical description of the nonbonded interactions in the molecular mechanics force fields, which use Lennard-Jones potential for the Pauli repulsion and long-range dispersion and the Coulomb potential for electrostatic interactions. Using this description, the potential energy as well as the forces between two particles go to infinity when the distance between the particles approaches zero. Numerical instabilities may arise during an alchemical transition when interatomic distance between atoms becomes very small and subsequently the force acting on the particles increases rapidly. In that case, a smaller time step would be required to avoid integration artifacts. To avoid singularities and numerical instabilities, Zacharias et al. ${ }^{27}$ proposed a method to scale and shift the Lennard-Jones potential such that at short distances the Lennard-Jones repulsion between two atoms converges to a finite value. Beutler et al. ${ }^{28}$ used a similar shifting for both the van der Waals and Coulombic interactions. Anwar and Heyes ${ }^{29}$ suggested a damping potential for the Ewald summation, which could be used with the soft-cored version of the Lennard-Jones potential. The version of the Beutler et al. ${ }^{28}$ soft-core potential is used in the Gromacs molecular dynamics package. ${ }^{30}$ Work by Boresch and Bruckner ${ }^{31}$ offers an alternative approach to the usual alchemical methods. Their way to solve the van der Waals end-state problem was not to describe the pathway between two states by a combination of Hamiltonians coupled to a parameter $\lambda$. Instead, the pathway was constructed from a series of atom insertions. Free energy differences between the discrete states then could be estimated using FEP or BAR formulas. For the thermodynamic integration scheme, however, the soft-core potential is still necessary.

Here, we show that, due to the nature of the current soft-core potential, unwanted minima in the nonbonded potential energy may occur for some combinations of Lennard-Jones and Coulomb parameters at intermediate $\lambda$ values, when attractive electrostatic interactions are not sufficiently counterbalanced by the van der Waals repulsion. We propose a new approach that prevents singularities and numerical instabilities and avoids the additional minima points during alchemical transitions. The new soft-core potential was validated by application to the free energy calculation of a closed thermodynamic cycle, the mutation influence on protein thermostability, the calculation of small ligand solvation free energies, and the estimation of binding free energies of trypsin inhibitors. The applications show that the new soft-core potential provides a robust and accurate general purpose solution to alchemical free energy calculations.

\section{THEORY AND METHODS}

2.1. Conventional Soft-Core Function. The commonly used soft-core potential function described by Beutler et al. ${ }^{28}$ occasionally suffers from additional minima points occurring during nonequilibrium transitions at intermediate $\lambda$ values, which manifest themselves as a sudden jump in $\partial H / \partial \lambda$, consequently strongly affecting the final free energy estimations. This effect is illustrated by an example in Figure 1, where we analyze an alchemical transition during which five atoms are introduced into the system. Consider a situation where atoms $\mathrm{C}, \mathrm{N}, \mathrm{O}, \mathrm{H}_{\mathrm{N1}}$, and $\mathrm{H}_{\mathrm{N} 2}$ are not present in the system in state $\mathrm{A}$. These atoms are appearing in the system in state $\mathrm{B}$ in a rapid nonequilibrium transition. The growth of the atoms is performed via a linear combination of Hamiltonians for both states using a coupling parameter $\lambda: H_{\lambda}=(1-\lambda) H_{A}+\lambda H_{B}$.

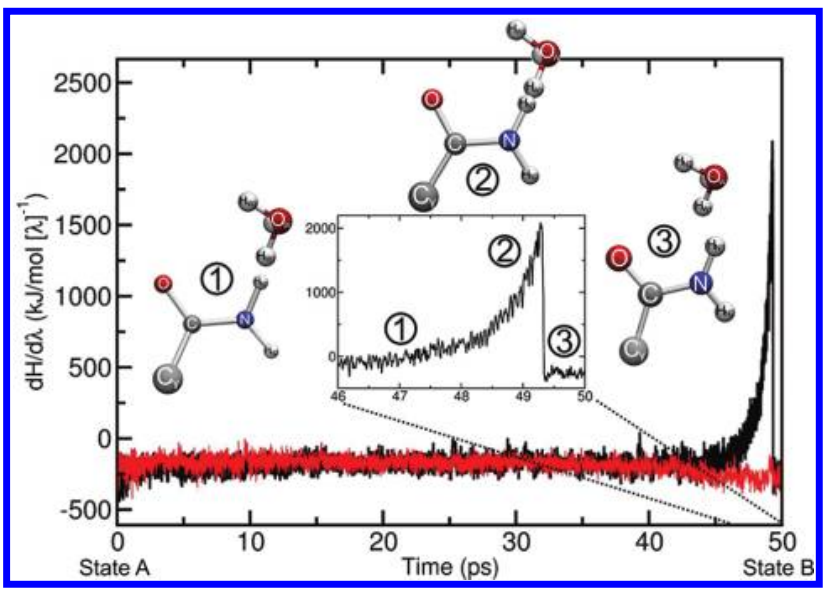

Figure 1. Origin of singularities in the $\partial H / \partial \lambda$ curves. The black curve illustrates a jump in $\partial H / \partial \lambda$, the red line shows a smooth behavior of the curve. The only difference between the two cases is the initial simulation structure, indicating that the jumps appear stochastically. The atoms $\mathrm{C}, \mathrm{N}, \mathrm{O}, \mathrm{H}_{\mathrm{N} 1}$, and $\mathrm{H}_{\mathrm{N} 2}$ are appearing in the system, whereas $\mathrm{C}_{\gamma}$ and the water molecule are present in the system throughout the transition. The Lennard-Jones parameters and partial charges of the atoms correspond to the OPLS force-field parameters; the water molecule is described by the TIP4P water model. State 1: water is attracted to the morphed atoms by an electrostatic interaction between the atoms $\mathrm{N}$ and $\mathrm{H}_{W 1}$ as well as $\mathrm{O}_{W}$ and $\mathrm{H}_{N 1}$. At this stage, the repulsive forces are weak. State 2: the water molecule remains close to the growing atoms for several picoseconds. During that time, electrostatic and Lennard-Jones repulsive forces are increasing, and the potential energy of the system is rising. State 3: repulsion overcomes attraction and the water molecule is expelled. The potential energy rapidly drops, creating a sharp peak in the $\partial H / \partial \lambda$. Molecular structures were visualized with VMD. ${ }^{58}$

Coulomb and Lennard-Jones interactions are turned on simultaneously; thus, a soft-core potential must be used. During the transition, a specific balance between electrostatic and Lennard-Jones interactions may result in a situation where a water molecule is likely to be attracted to the morphed atoms. Attractive electrostatic interaction between the atoms $\mathrm{N}$ and $\mathrm{H}_{W 1}$ as well as $\mathrm{O}_{W}$ and $\mathrm{H}_{N 1}$ cannot be compensated by the repulsive electrostatic interaction between the $\mathrm{N}$ and $\mathrm{O}_{W}$ atoms, which is not strong enough in this stage to push the water molecule away. Hydrogen atoms in the OPLS force field, ${ }^{32}$ which was used for this example, have zero LennardJones parameters. Due to the applied soft-core potential, the van der Waals repulsion between $\mathrm{N}$ and $\mathrm{O}_{W}$ atoms is weakened. Such an arrangement of the atoms can result in a water molecule remaining close to the growing atoms for several picoseconds due to the shape of the soft-cored energy curve (as will be explained further). During that time, electrostatic and Lennard-Jones repulsive forces are increasing and the potential energy of the system is rising until the point where repulsion overcomes attraction and the water molecule is expelled. At this point, the potential energy rapidly drops, creating a sharp peak in the $\partial H / \partial \lambda$ curve. We also demonstrate the occurrence of an additional minima by analyzing potential energy and force surfaces for two and four interacting atoms (see the Supporting Information, S5). This effect is not only observed in the OPLS force field. Similar jumps in the curves were also observed in the generalized amber force field (GAFF) ${ }^{33}$ parametrized ligand simulations. One way to solve this problem is to switch electrostatic and Lennard-Jones interactions separately. However, this approach would not only double or triple the 


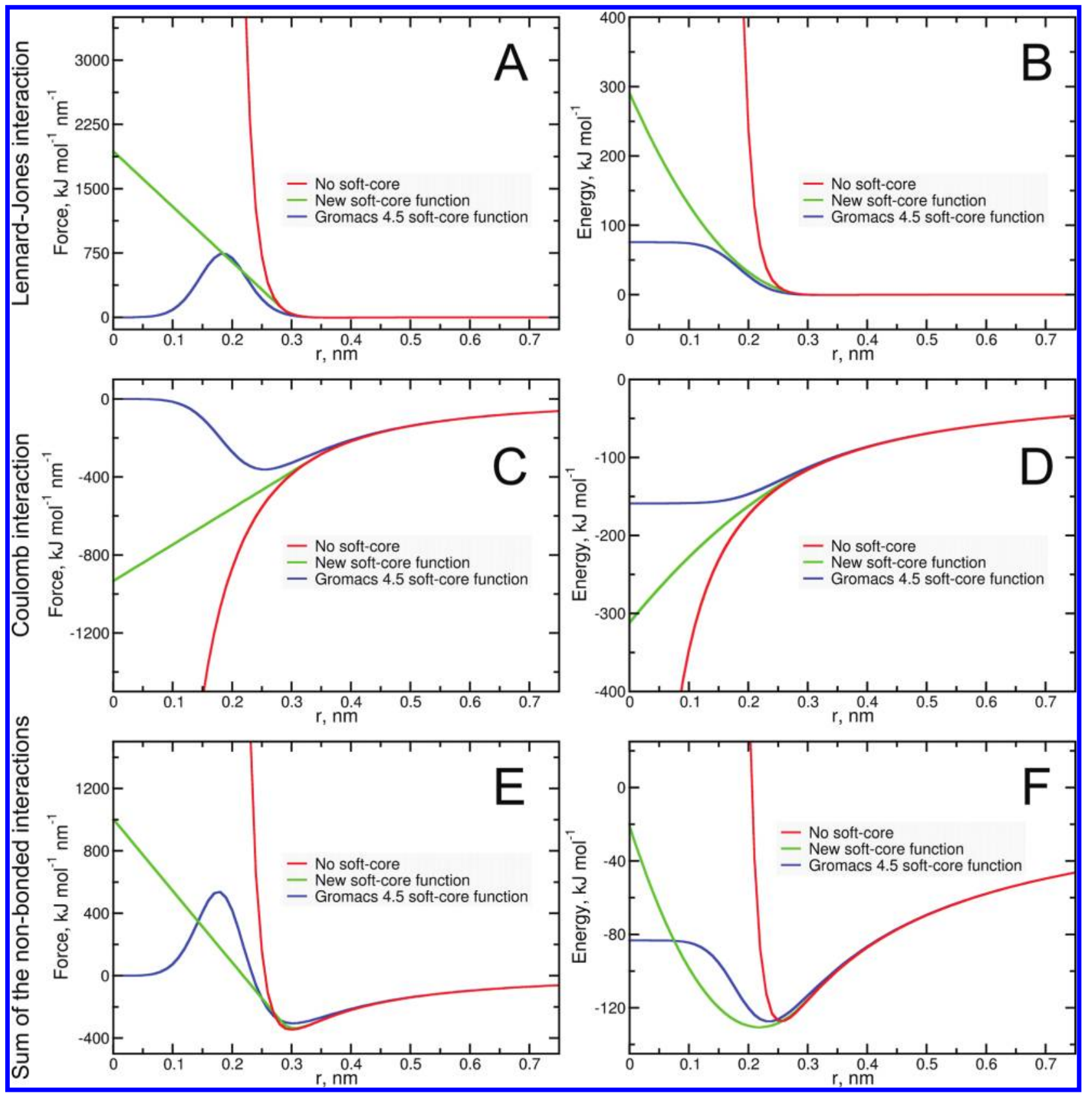

Figure 2. Force and potential curves for the nonbonded interactions between two atoms at $\lambda=0.5$. Both atoms in consideration were assigned identical Lennard-Jones parameters: $\sigma=0.3 \mathrm{~nm}$ and $\varepsilon=0.5 \mathrm{~kJ} / \mathrm{mol}$. Partial charges of the atoms were $q_{1}=0.5$ and $q_{2}=-0.5$. Gromacs 4.5 soft-core parameters: $\alpha=0.3$ and $\sigma=0.3$. Parameters for the new soft-core function: $\alpha_{L J}=0.85, \alpha_{Q}=0.3$ and $\sigma_{Q}=1$. (A) Lennard-Jones interaction force. (B) Lennard-Jones interaction energy. (C) Coulomb interaction force. (D) Coulomb interaction energy. (E) Total nonbonded interaction energy. (F) Total nonbonded interaction force.

simulation time, but it is also impracticable for a CFT setup in which equilibrium simulations of the end-states are required.

2.2. Origin of the Singularities. To avoid singularities and numerical instabilities during the appearance and annihilation of the particles in a system, Beutler et al. ${ }^{28}$ suggested "softening" the Lennard-Jones and Coulomb interactions (blue curves in the Figure 2B, D, F). The potential energy functions were modified, such that, instead of going to infinity at short distances, they reach a finite plateau value. The Gromacs implementation of the soft-core function follows a slightly modified Beutler soft-core potential. The nonbonded part of the potential function for the system in the state A can be written as follows: ${ }^{34}$

$$
V_{i j}\left(r_{i j}\right)=\frac{q_{i} q_{j}}{4 \pi \varepsilon_{0} \varepsilon_{\mathrm{r}} r_{\mathrm{A}}}+\left(\frac{C_{i j}^{(12)}}{r_{\mathrm{A}}^{12}}-\frac{C_{i j}^{(6)}}{r_{\mathrm{A}}^{6}}\right)
$$

where $r_{\mathrm{A}}=\left(r_{i j}^{6}+\alpha \sigma_{\mathrm{A}}^{6} \lambda^{p}\right)^{1 / 6}$ is the distance between the atoms $i$ and $j$ for the state A, $q_{i}$ and $q_{j}$ are partial charges of the atoms $i$ and $j, \varepsilon_{0}$ is the dielectric constant in a vacuum, $\varepsilon_{\mathrm{r}}$ is the relative dielectric constant, and $C_{i j}^{(12)}$ and $C_{i j}^{(6)}$ are the Lennard-Jones parameters. The charges $q_{i}, q_{j}$ and parameters $C_{i j}^{(12)}, C_{i j}^{(6)}$ usually are different for states $\mathrm{A}$ and $\mathrm{B}$; however, for the sake of simplicity we omit the state indices. $\alpha, p$, and $\sigma$ are parameters, where $\sigma=\left(C_{i j}^{(12)} / C_{i j}^{(6)}\right)^{1 / 6}$. If one of the Lennard-Jones parameters is equal to 0 , a user defined $\sigma$ value is used. For 
state $\mathrm{B}$, the distance between the two atoms is defined as $r_{\mathrm{B}}=$ $\left(r_{i j}^{6}+\alpha \sigma_{\mathrm{B}}^{6}(1-\lambda)^{p}\right)^{1 / 6}$.

The Gromacs soft-core potential implements only one parameter $\alpha$ for both the Lennard-Jones and Coulomb interactions, whereas the Beutler et $\mathrm{al}^{28}$ formulation allows controlling this parameter separately for the van der Waals interaction and electrostatics. Also, Gromacs implements only one of the parameter $s=p=6$ sets (following the notation of the eq 9 in Beutler et al. ${ }^{28}$ ). Hence, Gromacs uses a special case of the Beutler soft-core potential. In addition, Gromacs allows choosing the power of $\lambda$ to be 1 or 2 , which for the Beutler softcore was set to 2 .

This approach allows overlap of the atoms involved in an alchemical transition and avoids singularities and numerical instabilities. However, there is a remaining risk for additional minima, the origin of which lies in the shape of the force curves (blue curves in the Figure 2A, C, E). With a decreasing distance between two particles, the force rises similar to the case of the hard-core interaction. However, at a certain distance, a maximal force is reached. If the atoms get even closer, the force between them decreases, reaching zero at very short distances (see surface plots for the nonbonded interaction energy and force in the Supporting Information, S5). This shape of the force curves explains the jumps in the $\partial H / \partial \lambda$ curves (Figure 1): atoms that are very close to each other may exert a weaker repulsion onto each other than the attraction between the atoms that are further apart. An unwanted minimum in the nonbonded energy landscape is created in which two atoms remain at a close distance to each other. Such a situation becomes a trap for the alchemically modifiable atoms, which is abruptly escaped at higher or lower $\lambda$ values. In our analysis, we primarily tested various subsets of parameters for the original soft-core formulation. As shown in Supporting Information, Figure S4, however, the risk for additional minima remains as a result of the shape of the force curves (Figure 2).

2.3. New Soft-Core Function. The new soft-core function is constructed as a switch between the soft and hard-core nonbonded interactions. In our approach, modifications take place at the level of the force and the modified potential energy is derived from it. For large distances van der Waals and Coulomb forces are described in their classical hard-core terms. At a certain distance, $r_{i j}^{\mathrm{LJ}}$ for the Lennard-Jones and $r_{i j}^{Q}$ for the Coulomb interaction, forces are linearized, retaining the slope of the force at the switch point. The switching distance is dependent on $\lambda$, ensuring that, at the end points of the transition, the system is described completely by a hard-core potential. The expressions for the Lennard-Jones force and its switching point are

$$
\mathbf{F}_{i j}^{\mathrm{LJ}}\left(\mathbf{r}_{i j}\right)=\left\{\begin{array}{lll}
\left(\frac{12 C_{i j}^{(12)}}{r_{i j}^{13}}-\frac{6 C_{i j}^{(6)}}{r_{i j}^{7}}\right) \frac{\mathbf{r}_{i j}}{r_{i j}}, & \text { if } & r_{i j} \geq r_{i j}^{\mathrm{LJ}} \\
\frac{\mathrm{d} \mathbf{F}_{i j}^{\mathrm{LJ}}}{\mathrm{d} r_{i j}} r_{i j} r_{i j}^{\mathrm{LJ}} \\
r_{i j}
\end{array}\right.
$$

where the switching point between the soft and hard-core Lennard-Jones forces $r_{i j}^{\mathrm{LJ}}=\alpha_{L J}\left({ }^{26} /{ }_{7}\left(C_{i j}^{(12)} / C_{i j}^{(6)}\right) \lambda\right)^{1 / 6}$ for state A and $r_{i j}^{\mathrm{LJ}}=\alpha_{L J}\left({ }^{26} /{ }_{7}\left(C_{i j}^{(12)} / C_{i j}^{(6)}\right)(1-\lambda)\right)^{1 / 6}$ for state $\mathrm{B}$ and $r_{i j}$ is the distance between the atoms $i$ and $j$.

The parameter $\alpha_{\mathrm{LJ}}$ controls the position of the switching point between the soft-core and hard-core Lennard-Jones interactions. With $\alpha_{\mathrm{LJ}}=1$ and $\lambda=1$ for state $\mathrm{A}$ (or $\lambda=0$ for state B), the van der Waals force is linearized at the minimum point and, thus, is equal to zero for distances shorter than $r_{i j}^{L J}$.

The electrostatic force is described as

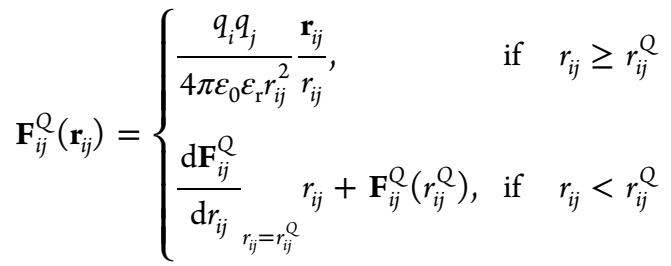

where the switching point between the soft and hard-core electrostatic forces $r_{i j}^{Q}=\left(1+\sigma_{Q}\left|q_{i} q_{j}\right|\right) \alpha_{Q} \lambda^{1 / 6}$ for state $\mathrm{A}$ and $r_{i j}^{Q}=$ $\left(1+\sigma_{Q}\left|q_{i} q_{j}\right|\right) \alpha_{Q}(1-\lambda)^{1 / 6}$ for state B.

$\alpha_{Q}$ is the control parameter for the switching point between the soft and hard-core electrostatic forces. $r_{i j}^{Q}$ also depends on the partial charges of the interacting atoms: larger absolute values of the charges result in a larger distance for the switching point, consequently making the interaction "softer". The influence of the charges on the switching point is controlled by the parameter $\sigma_{Q}$. The power $1 /{ }_{6}$ for $\lambda$ makes sure that the switching point for the Coulomb interaction changes at the same rate with respect to $\lambda$ as the switching point for the Lennard-Jones interaction.

The expression for the Lennard-Jones potential energy is

$$
V_{i j}^{\mathrm{LJ}}\left(r_{i j}\right)=\left\{\begin{array}{lll}
\frac{C_{i j}^{(12)}}{r_{i j}^{12}}-\frac{C_{i j}^{(6)}}{r_{i j}^{6}}, & \text { if } & r_{i j} \geq r_{i j}^{\mathrm{LJ}} \\
-\int_{r} \mathbf{F}_{i j}^{\mathrm{LJ}} \mathrm{d} \mathbf{r}_{i j}+C_{\mathrm{LJ}}, & \text { if } & r_{i j}<r_{i j}^{\mathrm{LJ}}
\end{array}\right.
$$

and that for the Coulomb potential energy is

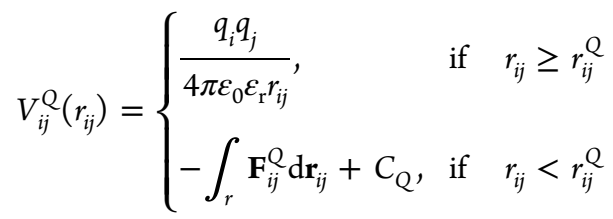

where $C_{\mathrm{LJ}}$ and $C_{Q}$ are integration constants.

$\partial H / \partial \lambda$ curves for distances larger than the switching point is simply equal to $V_{\mathrm{B}}-V_{\mathrm{A}}$. For the distances below the switching point

$$
\frac{\partial H}{\partial \lambda}=V_{\mathrm{B}}-V_{\mathrm{A}}+(1-\lambda) \frac{\partial V_{\mathrm{A}}}{\partial \lambda}+\lambda \frac{\partial V_{\mathrm{B}}}{\partial \lambda}
$$

Explicit expressions for the forces, potential energies and $\partial H / \partial \lambda$ curves can be found in the Supporting Information, S1. In the Supporting Information, S2, the newly constructed force and potential energy functions are shown to converge to the original formulation of the Lennard-Jones and Coulomb interactions at the switching point. The derivation of $\partial H / \partial \lambda$ is provided in the Supporting Information, S3.

The new soft-core function avoids additional extrema points in the force curves (green curves in the Figure 2A, C, E). The force between two particles monotonically decreases with an increase in distance in the soft-cored distance range. The potential energy functions (green curves in the Figure 2B, D, F) for the nonbonded interactions reach finite (but nonzero) values when the distance between particles goes to zero. This means that, during particle introduction/annihilation in the system, overlaps of the modifiable atoms are allowed, but 


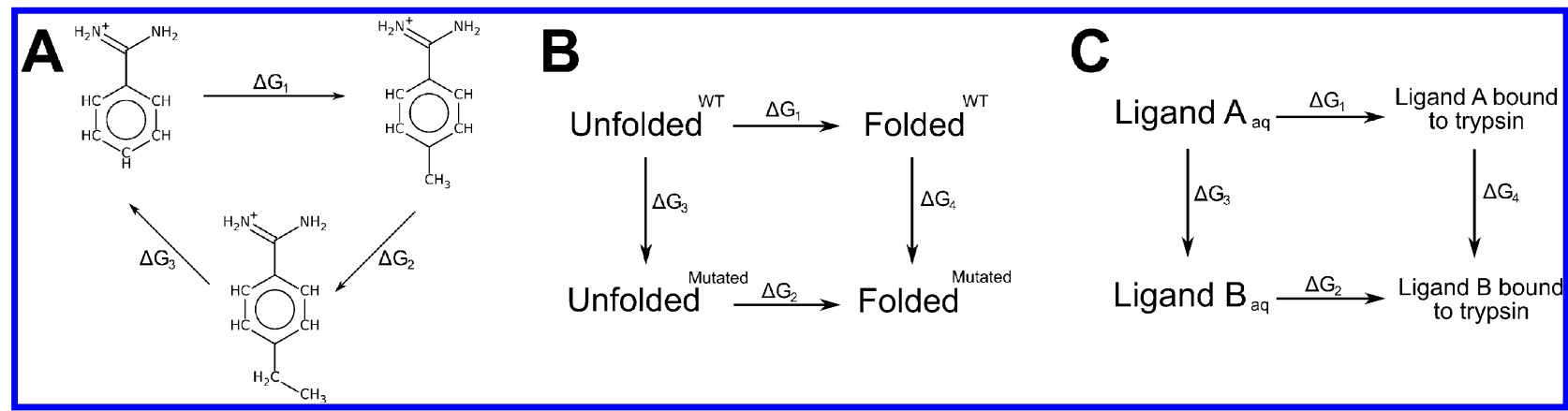

Figure 3. Schematic representations of the thermodynamic cycles. (A) Closed thermodynamic cycle. The free energy of the cycle is calculated by $\Delta G_{\text {cycle }}=\Delta G_{1}+\Delta G_{2}+\Delta G_{3}$, which should be zero by construction. (B) Thermodynamic cycle for the alanine mutations in the enzyme barnase. The relative free energy difference is calculated by $\Delta \Delta G=\Delta G_{1}-\Delta G_{2}=\Delta G_{3}-\Delta G_{4}$. (C) Thermodynamic cycle for trypsin inhibitors. Differences in free energies of binding between the ligands were calculated using the expression $\Delta \Delta G=\Delta G_{2}-\Delta G_{1}=\Delta G_{4}-\Delta G_{3}$. The $2 \mathrm{D}$ representation of the molecules was created with Marvin 5.3.8, 2010, ChemAxon (http://www.chemaxon.com).

clashes cause repulsion that gets stronger with decreasing distance. This construction avoids the traps inherent to the original soft-core.

At zero interparticle distance, that is, when two atoms completely overlap, the new formulation of the soft-core function is discontinuous. However, we show, both analytically and computationally (see the Supporting Information, S4), that a complete overlap represents an entropically forbidden state, which therefore does not affect the estimated free energies.

When using the new soft-core function, slight jumps in $\partial \mathrm{H} /$ $\partial \lambda$ are expected in the beginning and the end of an alchemical transition. As shown in the validation free energy calculations, these slight jumps have no influence on the free energy estimates. Additional smoothening of the $\partial H / \partial \lambda$ curves can be achieved by adjusting soft-core controlling parameters.

2.4. Parameter Selection. Selection of the optimal softcore parameter set is a nontrivial task. To obtain accurate free energy estimates, smooth $\partial H / \partial \lambda$ curves are desirable. Another requirement is to maintain van der Waals repulsion between two particles at short distances stronger than the electrostatic attraction to prevent atoms collapsing onto each other. However, as already shown in the example in Figure 1, the repulsion/attraction balance is a matter of interaction of many particles and considering only pairwise interactions would not be sufficient to calibrate the parameter set. In the new construction of the soft-core function, the parameter $\alpha_{\mathrm{LJ}}$ controls the point of linearization of the van der Waals interaction force. For $\alpha_{\mathrm{LJ}}$, the values range from 0 to $1: \alpha_{\mathrm{LJ}}=0$ implies a completely hard-core Lennard-Jones potential throughout the whole transition, $\alpha_{\mathrm{LJ}}=1$ would set the van der Waals force to $0 \mathrm{~kJ} \mathrm{~mol}^{-1} \mathrm{~nm}^{-1}$ at short distances for the end states. Values larger than 1 for this parameter would result in an attractive force at short distances, hence such values are not reasonable. Setting $\alpha_{\mathrm{LJ}}$ to be closer to 1 smooths the $\partial H / \partial \lambda$ curves. In our simulations, we chose $\alpha_{\mathrm{LJ}}=0.85$.

The value for the $\alpha_{Q}$ parameter controls the point of linearization of the electrostatic force. Since the Coulomb force curve does not have an extremum point, there is no caveat of selecting a value for $\alpha_{\mathrm{Q}}$, which would change the repulsion into attraction, or the other way around, as it was for the van der Waals interaction. Smaller $\alpha_{Q}$ values bring the electrostatic interaction closer to the original hard-core Coulomb potential, and the $\partial H / \partial \lambda$ curves become smoother. However, if an electrostatic potential becomes too similar to the classical Coulomb potential, the risk of singularities, as described by Anwar, ${ }^{29}$ increases. Larger values of $\alpha_{Q}$ may produce jumps in $\partial H / \partial \lambda$ in the beginning and the end of each transition. Since these jumps are predictable and occur in every transition, their influence on the final free energy estimate cancels. To avoid additional minima points and to smoothen the $\partial H / \partial \lambda$ curves, testing of the new soft-core function guided the choice for the $\alpha_{Q}$ to be about the van der Waals radius of the largest atom in the system. For the validation simulations, we used $\alpha_{Q}=0.3$. The switching point for the linearization of the electrostatics also depends on the partial charges of the atoms interacting: the larger charges the atoms carry, the more soft-cored their electrostatic interaction should be. This effect can be controlled with the parameter $\sigma_{Q}$, which in our simulations we simply set to be equal to 1 (details concerning the $\sigma_{Q}$ parameter are provided in the Supporting Information, S9). More information on the validity assessment for the selected parameter set as well as on the behavior of the forces and potentials depending on the parameters is provided in the Supporting Information, S6 and S7.

Parameters used for the Gromacs soft-core implementation were the following: $\alpha=0.3, \sigma=0.25, p=1$.

2.5. Simulation Details. In our approach, free energies were estimated from the nonequilibrium simulations relying on the Crooks Fluctuation Theorem. The nonequilibrium transition runs need to be started from structures taken from an equilibrium ensemble. Equilibrium simulations were performed for $10 \mathrm{~ns}$ at the states $\lambda=0$ and $\lambda=1$ using a stochastic dynamics integrator. From the last $8 \mathrm{~ns}$ of these simulations, 100 snapshots were extracted and fast nonequilibrium transitions were performed for 50 ps each using a leapfrog integrator. A time step of 2 fs was used for all the simulations. For the simulations of the barnase mutations in the Amber99 $\mathrm{sb}^{35}$ force-field, equilibrium ensembles generated by Seeliger and de Groot $^{16}$ were used. The work values for the forward and backward transitions were calculated using eq 2 . The Crooks Gaussian Intersection ${ }^{36}$ method was used to estimate free energy differences.

The new soft-core function was validated by calculating free energies of a closed thermodynamic cycle, changes in the free energy due to the mutations of the enzyme barnase, solvation free energies of small organic compounds, and ligands binding affinities to the enzyme trypsin. For the closed cycle, deviations from the expected value of zero were monitored. For the other cases, root-mean-square differences (RMSD) between the computed and experimental values were calculated. RMSDs retrieved using the new soft-core function were compared to the RMSDs calculated with the soft-core function implemented 
in Gromacs 4.5. For the barnase mutations in the Amber99sb force-field, simulations with the Gromacs 4.5 soft-core were not performed, but the results from Seeliger and de Groot, who used Gromacs 4.0 with the Beutler et al. ${ }^{28}$ type soft-core, were considered for comparison. All the simulations involving the new soft-core function were carried out with Gromacs 4.0.

Standard deviations for the calculated free energies were estimated, as described by Goette and Grubmüller. ${ }^{36}$

Closed Cycle. A closed thermodynamic cycle was constructed by morphing benzamidinium derivatives, as shown in Figure 3A. In the first step, a hydrogen on the benzene ring was morphed into a methyl group; in the second step, methyl was transformed into ethyl. The cycle was closed by morphing ethyl back to hydrogen. The free energy difference of traveling over the closed cycle was evaluated by $\Delta G_{\text {cycle }}=\Delta G_{1}+\Delta G_{2}+\Delta G_{3}$, which should be zero by construction. $\Delta G_{\text {cycle }}$ was calculated for various numbers of transitions in order to assess the convergence for the different soft-core functions. The simulations were prepared by putting each ligand in a dodecahedron box with at least $1.2 \mathrm{~nm}$ distance from the solute to a box wall. Ligands were solvated with TIP $3 \mathrm{P}^{37}$ water, resulting in around 800 water molecules in a box. $\mathrm{Na}^{+}$and $\mathrm{Cl}^{-}$ ions were added to neutralize the system and reach $150 \mathrm{mM}$ salt concentration. The Amber99sb force field was used for the simulations; topology parameters for the ligands were retrieved using GAFF. Gaussian $03^{38}$ was used to calculate partial charges. Temperature coupling was performed with the velocity rescaling thermostat ${ }^{39}$ with a time constant of $0.1 \mathrm{ps}$ and a reference temperature of $298 \mathrm{~K}$. The Parrinello-Rahman barostat $^{40}$ with a time constant of 1 ps was used to maintain a pressure of 1 bar. A cutoff of $1.1 \mathrm{~nm}$ for the Lennard-Jones potential with a switch function starting at $1 \mathrm{~nm}$ was used. The switch function for the van der Waals interactions that we used in all the simulations is acting at the force level, allowing for a smooth transition to $0 \mathrm{~kJ} \mathrm{~mol}^{-1} \mathrm{~nm}^{-1}$ in the region defined by the switching cutoff and the Lennard-Jones cutoff. The relevant equations for the switch function implemented in Gromacs can be found in a work by van der Spoel and van Maaren ${ }^{41}$ and the Gromacs User Manual. ${ }^{34}$ Dispersion was corrected for energy and pressure. Short-range electrostatic interactions were cutoff at $1.2 \mathrm{~nm}$. Particle Mesh Ewald (PME) ${ }^{42,43}$ was used for the long-range electrostatic interactions with the Fourier grid spacing set to $0.14 \mathrm{~nm}$ and interpolation of order 4 . Bonds and angles of water molecules were constrained using SETTLE. ${ }^{44}$ LINCS $^{45}$ was used to constrain all bonds in the ligands.

Barnase Mutations. To investigate the influence of mutations on the thermostability of the microbial ribonuclease barnase (PDB id 1BNI), alanine at position 32 was mutated into 14 different amino acids. Free energy differences caused by an alanine mutation were calculated in both the folded and the unfolded states of barnase. The unfolded state of the protein was approximated by using tripeptides GXG, where $\mathrm{X}$ was the mutated residue. Relative free energy differences were estimated from the thermodynamic cycle shown in Figure 3B: $\Delta \Delta G=\Delta G_{1}-\Delta G_{2}=\Delta G_{3}-\Delta G_{4}$. Simulations were carried out using two different force fields: Amber99sb and OPLS. The simulation set up was analogous to that described by Seeliger and de Groot. ${ }^{16}$ A switch function was used for the van der Waals interactions between 1.0 and $1.1 \mathrm{~nm}$. Electrostatics were treated with PME using a real space cutoff of $1.2 \mathrm{~nm}$, a Fourier grid spacing of $0.14 \mathrm{~nm}$, and an interpolation of order 4. Topologies for the mutated residues were generated using PYMACS (http://wwwuser.gwdg.de/ dseelig/pymacs.html). For the simulations with the Amber99sb, systems were solvated with TIP3P water, whereas the TIP4P water model was chosen for the simulations using the OPLS force field. Estimated free energy differences were compared to the experimental values obtained by Horovitz et al. ${ }^{46}$

Free Energies of Ligand Solvation. A set of 17 compounds preselected and analyzed by Nicholls et al. ${ }^{13}$ was chosen for a further validation of the new soft-core function by calculating free energies of ligand solvation. During the alchemical transitions, nonbonded interactions between a ligand and the solvent were switched on/off while intramolecular nonbonded interactions for the ligand were retained. The simulation system was set up by putting each ligand in a cubic box with dimensions of $2.5 \times 2.5 \times 2.5 \mathrm{~nm}$. The TIP3P water model was used to solvate the ligands, resulting in $\approx 500$ water molecules in a box. Topology parameters for the ligands were taken from GAFF. AM1-BCC $\mathrm{v1}^{47,48}$ partial charges provided by Nicholls et al. were used. All the set up parameters were set to be the same as those used by Nicholls et al. and described by Mobley et al., ${ }^{12}$ except the following: a neighbor list of $0.9 \mathrm{~nm}$ was used and both the equilibrium runs and the nonequilibrium transitions were performed using Berendsen pressure coupling algorithm ${ }^{49}$ with a reference pressure set to $1.0 \mathrm{bar}$ and time constant 0.5 ps. Following Nicholls et al., we set the van der Waals interactions to switch off between 0.8 and $0.9 \mathrm{~nm}$, the Coulomb real space cutoff was set to $0.9 \mathrm{~nm}$, and PME electrostatics treatment was employed with an interpolation of order 6 and a grid spacing of $0.1 \mathrm{~nm}$. For the nonequilibrium transition runs, a velocity rescaling thermostat with a time constant $0.1 \mathrm{ps}$ and reference temperature $300 \mathrm{~K}$ was used. The results were compared to the free energies estimated by Nicholls et al. using alchemical methods with the Merck-Frosst AM1-BCC v1 partial charges. The experimental values of the solvation free energies were selected by Nicholls et al. from refs 50-55.

Trypsin Inhibitors. For the protein-ligand binding free energy estimation, a set of trypsin binders described and tested by Talhout et al. ${ }^{56}$ was selected. Simulations of the ligands in water and in the active site of the enzyme were performed, and the relative free binding energies were estimated following the thermodynamic cycle in Figure 3C: $\Delta \Delta G=\Delta G_{2}-\Delta G_{1}=\Delta G_{4}$ $-\Delta G_{3}$. To prepare ligands in solution and to generate the topologies, the same protocol as that used for the ligands in the closed cycle simulations was followed. For the simulations of the ligands in the active site of the enzyme, the trypsin X-ray structure (PDB id 3PTB) was chosen. The ligand and trypsin were put in a dodecahedron box with at least $1.2 \mathrm{~nm}$ distance from a box wall. The system was solvated with TIP3P water resulting in $\approx 9000$ water molecules in the box. Ions were added to neutralize the system and mimick $150 \mathrm{mM}$ salt concentration. Simulations were carried out in the Amber99sb force field; GAFF was used for ligand parametrization. The molecular dynamics parameters were identical to those used in the closed cycle simulations.

\section{RESULTS}

3.1. Closed Cycle. The results of the free energy difference calculations for the closed thermodynamic cycle are shown in Figure 4. For both cases, Gromacs 4.5 and the new soft-core, the sum of three free energy differences across the closed cycle converges to zero as the number of transitions increases. Calculations using the soft-core function implemented in Gromacs 4.5 converge to $\approx 0 \mathrm{~kJ} / \mathrm{mol}$ already after 50 


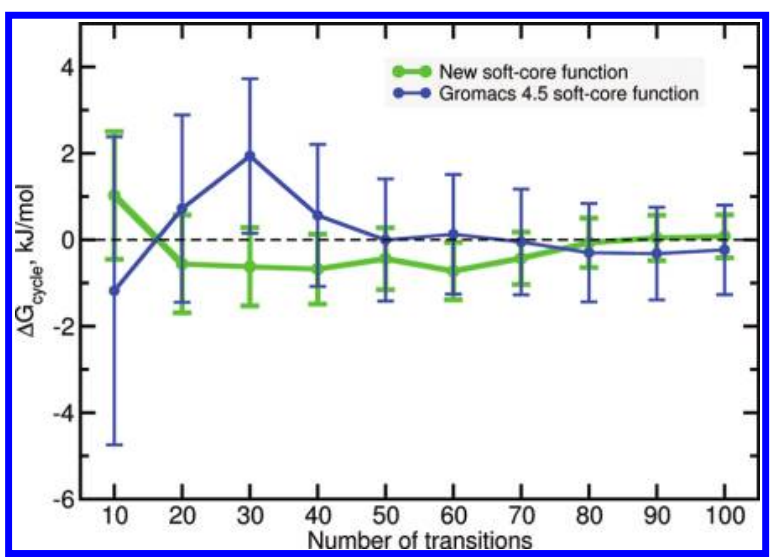

Figure 4. Free energy difference over a closed thermodynamic cycle. The $X$-axis denotes the number of transitions considered for the calculation of the free energies. Error bars represent standard errors. The dashed line marks the expected $0 \mathrm{~kJ} / \mathrm{mol}$.

transitions; however, with the further increase in the number of transitions $\Delta G_{\text {cycle }}$ starts slightly deviating from zero. The new soft-core function converges to zero after 70 transitions and stays at that value. After 100 transitions Gromacs 4.5 soft-core showed the value of $-0.24 \pm 1.03 \mathrm{~kJ} / \mathrm{mol}$, whereas the new soft-core ended up in $0.08 \pm 0.50 \mathrm{~kJ} / \mathrm{mol}$ for the closed cycle. The statistical accuracy for the closed thermodynamic cycle calculations with the new-soft core function is approximately two times higher in comparison to the calculations with the Gromacs 4.5 soft-core function.

3.2. Barnase Mutations. The influence of the soft-core potential function used for the free energy calculations of the barnase mutations was assessed for two force fields, Amber99sb and OPLS (Figure $5 \mathrm{~A}$ and $\mathrm{B}$, respectively). In the simulations with the Amber99sb force-field, 10 out of 14 calculated free energy values were closer to the experimentally measured values when the new soft-core function was used. Average RMSD between free energies calculated by Seeliger and de Groot with the Gromacs 4.0 soft-core and the experimental values was $1.61 \pm 0.20 \mathrm{~kJ} / \mathrm{mol}$, whereas for the new soft-core average RMSD was $1.35 \pm 0.15 \mathrm{~kJ} / \mathrm{mol}$. For the OPLS forcefield, free energies for 8 out of 14 amino acid mutations were more accurate when the new soft-core was used. Average
RMSD for the Gromacs 4.5 soft-core was $2.80 \pm 0.22 \mathrm{~kJ} / \mathrm{mol}$ and for the new soft-core $2.60 \pm 0.17 \mathrm{~kJ} / \mathrm{mol}$. When comparing the performance of two force-fields, free energies calculated in the Amber99sb in eight cases are closer to the experimental values than the calculations with the OPLS for the new softcore potential. For five mutations, the free energy estimation with the new soft-core in the OPLS force-field deviates from the experimental results by more than $1 \mathrm{kcal} / \mathrm{mol}$, whereas Amber99sb estimations are within $1 \mathrm{kcal} / \mathrm{mol}$ from the experimental values in all 14 cases.

3.3. Free Energies of Ligand Solvation. Solvation free energies were calculated for 17 small organic compounds. Results of the calculations were compared to the experimental values provided in the work of Nicholls et al. (Figure 6). For

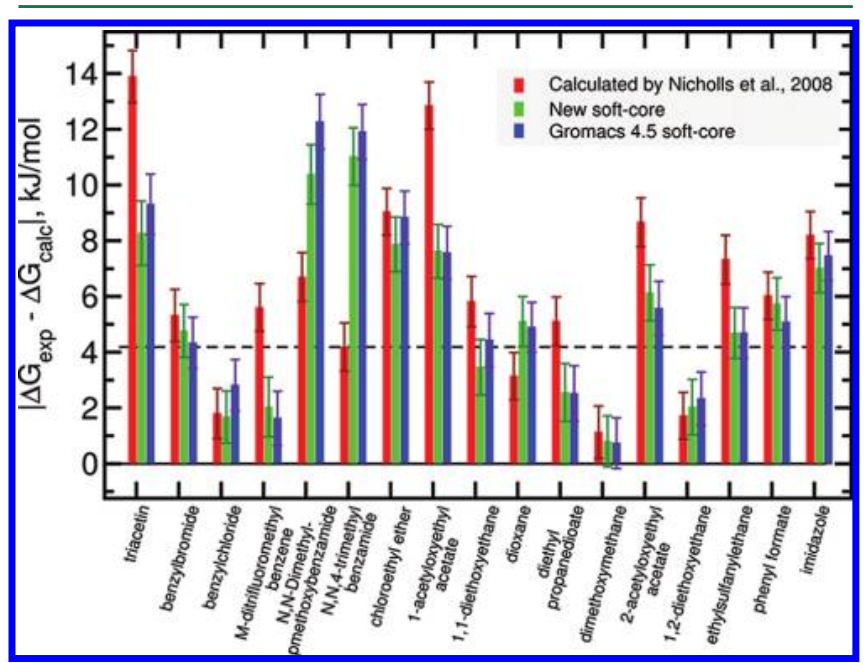

Figure 6. RMSD of the solvation free energies from the experimental values. ${ }^{13,50-55}$ Error bars represent standard errors. The dashed line marks a $1 \mathrm{kcal} / \mathrm{mol}$ deviation.

nine compounds, the new soft-core function performed better than the Gromacs 4.5 soft-core. When compared to the discrete TI calculations performed by Nicholls et al., 13 out of 17 solvation free energies were predicted more accurately by our nonequilibrium approach with the new soft-core function. Average RMSDs between calculated and experimentally

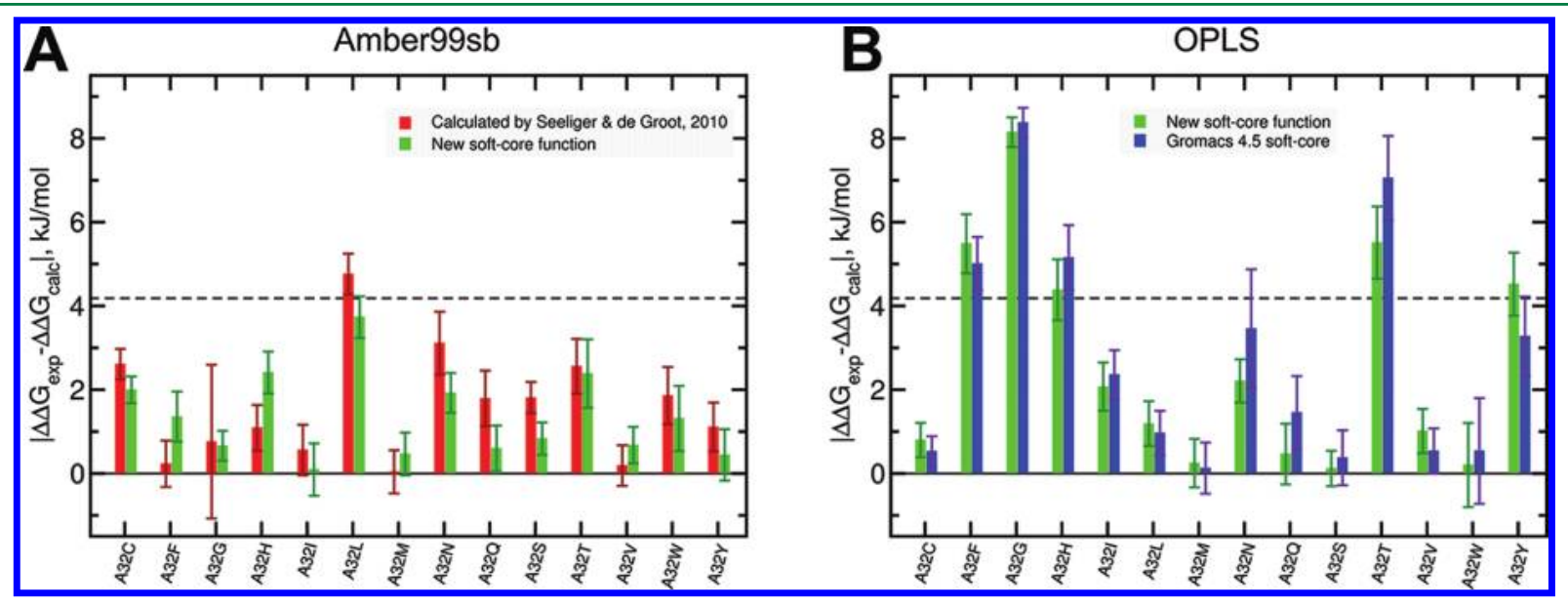

Figure 5. RMSD of the alanine mutation associated free energy changes in barnase from the experimental values. ${ }^{46}$ (A) Results of the simulations in the Amber99sb force field. (B) Results using the OPLS force field. Error bars represent standard errors. The dashed line marks a $1 \mathrm{kcal} / \mathrm{mol}$ deviation. 
obtained free energies are $6.26 \pm 0.21 \mathrm{~kJ} / \mathrm{mol}$ for the Nicholls et al. calculations, $5.36 \pm 0.24 \mathrm{~kJ} / \mathrm{mol}$ for the new soft-core, and $5.67 \pm 0.23 \mathrm{~kJ} / \mathrm{mol}$ for the Gromacs 4.5 soft-core function.

3.4. Trypsin Inhibitors. Free energies of binding were calculated for seven trypsin inhibitors described by Talhout et al. $^{56}$ (Figure 7). In five cases, calculations with the new soft-

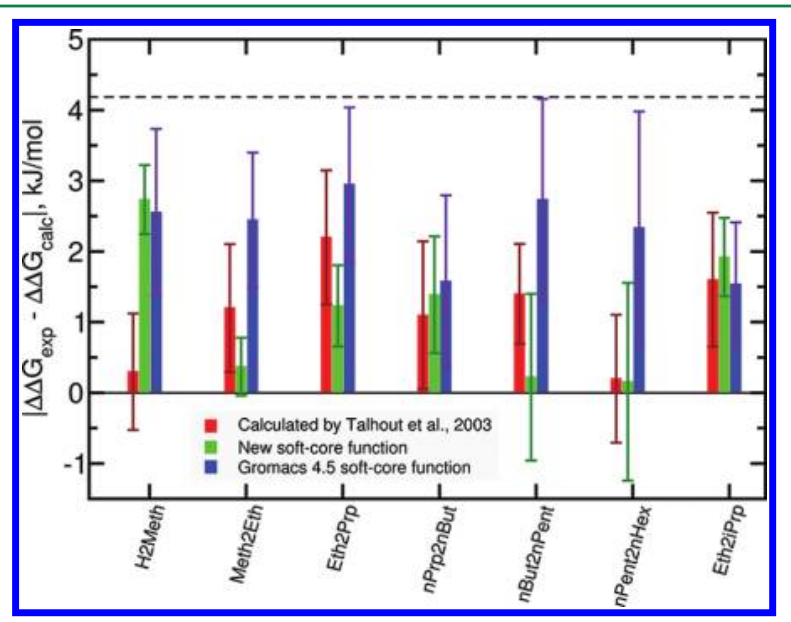

Figure 7. RMSD of the trypsin inhibitors binding free energies from the experimental values. Error bars represent standard errors. The dashed line marks a $1 \mathrm{kcal} / \mathrm{mol}$ deviation.

core function yielded more accurate results than the Gromacs 4.5 soft-core. Average RMSDs for the trypsin inhibitors are 1.14 $\pm 0.34 \mathrm{~kJ} / \mathrm{mol}$ for Talhout et al. ${ }^{56}$ calculations, $1.15 \pm 0.32 \mathrm{~kJ} /$ mol for the new soft-core function, and $2.31 \pm 0.46 \mathrm{~kJ} / \mathrm{mol}$ for the Gromacs 4.5 soft-core potential.

\section{DISCUSSION}

The free energy calculation for the closed cycle served as a robust and simple validation case for the new soft-core function. The expected outcome of this test $(0 \mathrm{~kJ} / \mathrm{mol})$ was reached using both constructions of the soft-core potential indicating correctness of both approaches. The larger error bars and slight divergence from zero for the Gromacs 4.5 soft-core can be attributed to minor jumps in the $\partial H / \partial \lambda$ curves observed during the simulations of the transitions in the closed cycle.

The calculation of free energies for barnase in the Amber99sb force field was intended as another check for the correctness of the new soft-core function. Calculations with the Gromacs 4.0 soft-core function showed no jumps in $\partial H / \partial \lambda$ in the simulations performed by Seeliger and de Groot. Therefore, it is interesting to notice that even in this case where the original soft-core function performs properly, calculations with the new soft-core function yield more accurate results. For the OPLS force field, the new soft-core also gives more accurate free energy estimates. For the mutations where the jumps in $\partial H / \partial \lambda$ were observed (Asn, Gln, Ser, Thr, Trp an Tyr) during the transitions with the Gromacs 4.5 soft-core potential, no jumps occurred when the new soft-core function was used. For all these cases, except tyrosine, free energy estimates were more accurate once the jumps in the curves were eliminated.

Concerning the tyrosine mutation, we analyzed the behavior of the $\partial H / \partial \lambda$ curves in more detail and discovered that, in cases where the original soft-core potential was used, the jumps in the curves occurred in the part of the thermodynamic cycle (Figure $3 \mathrm{~B})$ where the transitions in the unfolded form of the protein took place (data not shown). Integration over the curves containing jumps influenced the final $\Delta \Delta G$ such that it appeared to be closer to the experimentally observed value. This result exemplifies the danger of the jumps in the $\partial H / \partial \lambda$ curves during the alchemical transitions: the results may appear to be more accurate due to an accidental trap for the modifiable atoms, as explained in Figure 1. Situations such as this one limit the predictive power and reliability of alchemical free energy methods.

The performance of the new soft-core function in the calculations of the ligand solvation free energies and binding affinities of the trypsin inhibitors is of particular importance because of the applicability of the alchemical methods in rational drug design. In spite of the fact that no jumps in the $\partial H / \partial \lambda$ curves were observed for the ligand solvation simulations with the Gromacs 4.5 soft-core, results obtained with new soft-core are more accurate. Also, the nonequilibrium free energy calculations appear to be superior to the discrete TI used by Nicholls et al. Application of the new soft-core potential for the binding free energy calculations of the trypsin inhibitors has a significant effect on the accuracy of the binding affinity estimates. Removal of the jumps in the curves reduced the RMSD to the experimental values in the free energy estimations by a factor of 2 , on average.

In some cases, the usage of the original soft-core does not cause entrapment in the additional minima, hence, no jumps in the $\partial H / \partial \lambda$ occur. For such cases, our aim was to illustrate that the new soft-core potential was providing consistent results with the original soft-core calculations. In other cases, such as trypsin inhibitor analysis, removal of the jumps in the $\partial H / \partial \lambda$ curves increased the accuracy of the free energy estimates. Lawrenz et al. ${ }^{57}$ demonstrated how additional sampling using independent $\mathrm{MD}$ runs, dramatically increased accuracy of the absolute free energy of binding estimation. In this context, improvements of using the new soft-core potential may be used in combination with additional sampling to enhance the accuracy of the free energy calculations.

Accurate free energy estimates and predictable behavior of the $\partial H / \partial \lambda$ curves make the nonequilibrium alchemical free energy calculations in combination with the new soft-core function an attractive method for the design of new drug candidates and ligand optimization. In addition, the new softcore potential can be applied to the equilibrium free energy calculations that may also suffer from additional minima when the original soft-core is applied.

\section{CONCLUSIONS}

We have introduced a novel soft-core potential for nonbonded interactions in alchemical free energy simulations, that alleviates additional minima in the nonbonded potential energy that may occur with the original soft-core formulation. The new soft-core function works as a switch between the traditional (hard-core) description of the nonbonded interactions and the linearized form of the Coulomb and Lennard-Jones forces. The switching point for the force linearization in the new soft-core function is $\lambda$ dependent, ensuring that the end states of a transition are described by classical hard-core potentials. The new soft-core function was validated by calculating the free energy over a closed thermodynamic cycle, free energy differences caused by mutations in an enzyme barnase in the Amber99sb and OPLS force fields, solvation free energies of small organic compounds, and free binding energies of trypsin inhibitors. In all cases, the new soft-core function leads to more accurate free energy estimates. 


\section{ASSOCIATED CONTENT}

\section{S Supporting Information}

S1: explicit expressions for the forces, potential energies, and $\partial H / \partial \lambda$ curves. S2: switching of the force and potential in the new soft-core shown to be continuous. S3: derivation of $\partial H / \partial \lambda$. S4: estimation of the probability of having zero distance between particles. S5: occurrence of additional minima caused by the soft-core. S6: parameter selection. S7: dependence of the new soft-core on the parameter set used. S8: behavior of Gromacs 4.5 and new soft-core as a function of $\lambda$. S9: importance of the parameter $\sigma_{\mathrm{Q}}$. S10, S11, and S12: tables containing experimental and calculated free energies for the barnase mutations, ligand solvation and trypsin inhibitors, respectively. This material is available free of charge via the Internet at http://pubs.acs.org/.

\section{AUTHOR INFORMATION}

\section{Corresponding Author}

*E-mail: bgroot@gwdg.de.

\section{Notes}

The authors declare no competing financial interest.

\section{ACKNOWLEDGMENTS}

V.G. was supported by an International Max Planck Research School for Physics of Biological and Complex Systems stipend.

\section{REFERENCES}

(1) Lamb, M. L.; Jorgensen, W. L. Curr. Opin. Chem. Biol. 1997, 1, 449-457.

(2) Jorgensen, W. L. Science 2004, 303, 1813-1818.

(3) Shirts, M. R.; Mobley, D. L.; Chodera, J. D. Annu. Rep. Comput. Chem. 2007, 3, 41-59.

(4) Christ, C. D.; Mark, A. E.; van Gunsteren, W. F. J. Comput. Chem. 2010, 31, 1569-1582.

(5) Villa, A.; Zangi, R.; Pieffet, G.; Mark, A. E. J. Comput.-Aided Mol. Des. 2003, 17, 673-686.

(6) Mobley, D. L.; Chodera, J. D.; Dill, K. A. J. Chem. Phys. 2006, $125,084902$.

(7) Mobley, D. L.; Graves, A. P.; Chodera, J. D.; McReynolds, A. C.; Shoichet, B. K.; Dill, K. A. J. Mol. Biol. 2007, 371, 1118-1134.

(8) Steiner, D.; Oostenbrink, C.; Diederich, F.; Zürcher, M.; van Gunsteren, W. F. J. Comput. Chem. 2011, 32, 1801-1812.

(9) Gago, F. Curr. Med. Chem.: Anti-Cancer Agents 2004, 4, 401-403.

(10) Alonso, H.; Bliznyuk, A. A.; Gready, J. E. Med. Res. Rev. 2006, 26, 531-568.

(11) Chodera, J. D.; Mobley, D. L.; Shirts, M. R; Dixon, R. W.; Branson, K.; Pande, V. S. Curr. Opin. Struct. Biol. 2011, 150-160.

(12) Mobley, D. L.; Dumont, É.; Chodera, J. D.; Dill, K. A. J. Phys. Chem. B 2007, 111, 2242-2254.

(13) Nicholls, A.; Mobley, D. L.; Guthrie, J. P.; Chodera, J. D.; Bayly, C. I.; Cooper, M. D.; Pande, V. S. J. Med. Chem. 2008, 51, 769-779.

(14) Mobley, D. L.; Bayly, C. I.; Cooper, M. D.; Shirts, M. R.; Dill, K. A. J. Chem. Theory Comput. 2009, 5, 350-358.

(15) Shivakumar, D.; Williams, J.; Wu, Y.; Damm, W.; Shelley, J.; Sherman, W. J. Chem. Theory Comput. 2010, 6, 1509-1519.

(16) Seeliger, D.; De Groot, B. L. Biophys. J. 2010, 98, 2309-2316.

(17) Åqvist, J.; Luzhkov, V. Nature 2000, 404, 881-884.

(18) Zwanzig, R. W. J. Chem. Phys. 1954, 22, 1420-1426.

(19) Lu, N.; Kofke, D. A. J. Chem. Phys. 2001, 114, 7303-7311.

(20) Lu, N.; Kofke, D. A. J. Chem. Phys. 2001, 115, 6866-6875.

(21) Bennett, C. J. Comput. Phys. 1976, 22, 245-268.

(22) Bruckner, S.; Boresch, S. J. Comput. Chem. 2011, 32, 13031319.

(23) Kirkwood, J. G. J. Chem. Phys. 1935, 3, 300-313.

(24) Jarzynski, C. Phys. Rev. Lett. 1997, 78, 2690-2693.
(25) Jarzynski, C. Phys. Rev. E: Stat. Phys., Plasmas, Fluids, Relat. Interdiscip. Top. 1997, 56, 5018-5035.

(26) Crooks, G. E. J. Stat. Phys. 1998, 90, 1481-1487.

(27) Zacharias, M.; Straatsma, T. P.; McCammon, J. A. J. Chem. Phys. 1994, 100, 9025-9031.

(28) Beutler, T. C.; Mark, A. E.; van Schaik, R. C.; Gerber, P. R.; van Gunsteren, W. F. Chem. Phys. Lett. 1994, 222, 529-539.

(29) Anwar, J.; Heyes, D. M. J. Chem. Phys. 2005, 122, 22411.

(30) Hess, B.; Kutzner, C.; van der Spoel, D.; Lindahl, E. J. Chem. Theory Comput. 2008, 4, 435-447.

(31) Boresch, S.; Bruckner, S. J. Comput. Chem. 2011, 32, 24492458.

(32) Jorgensen, W. L.; Maxwell, D. S.; Tirado-Rives, J. J. Am. Chem. Soc. 1996, 118, 11225-11236.

(33) Wang, J.; Wolf, R. M.; Caldwell, J. W.; Kollman, P. A.; Case, D. A. J. Comput. Chem. 2004, 25, 1157-1174.

(34) van der Spoel, D.; Lindahl, E.; Hess, B.; van Buuren, A. E.; Apol, E.; Meulenhoff, P. J.; Tieleman, D. P.; Sijbers, A. L. T. M.; Feenstra, K. A.; van Drunen, R; Berendsen, H. J. C. Gromacs User Manual, Version 4.5; GROMACS Development Team: Uppsala University, Sweden, 2010.

(35) Hornak, V.; Abel, R.; Okur, A.; Strockbine, B.; Roitberg, A.; Simmerling, C. Proteins Struct. Funct. Bioinf. 2006, 65, 712-725.

(36) Goette, M.; Grubmüller, H. J. Comput. Chem. 2009, 30, 447456.

(37) Jorgensen, W. L.; Chandrasekhar, J.; Madura, J. D.; Impey, R. W.; Klein, M. L. J. Chem. Phys. 1983, 79, 926-935.

(38) Frisch, M. J.; Trucks, G. W.; Schlegel, H. B.; Scuseria, G. E.; Robb, M. A.; Cheeseman, J. R.; Montgomery, J. A., Jr.; Vreven, T.; Kudin, K. N.; Burant, J. C.; Millam, J. M.; Iyengar, S. S.; Tomasi, J.; Barone, V.; Mennucci, B.; Cossi, M.; Scalmani, G.; Rega, N.; Petersson, G. A.; Nakatsuji, H.; Hada, M.; Ehara, M.; Toyota, K.; Fukuda, R.; Hasegawa, J.; Ishida, M.; Nakajima, T.; Honda, Y.; Kitao, O.; Nakai, H.; Klene, M.; Li, X.; Knox, J. E.; Hratchian, H. P.; Cross, J. B.; Bakken, V.; Adamo, C.; Jaramillo, J.; Gomperts, R.; Stratmann, R. E.; Yazyev, O.; Austin, A. J.; Cammi, R.; Pomelli, C.; Ochterski, J. W.; Ayala, P. Y.; Morokuma, K.; Voth, G. A.; Salvador, P.; Dannenberg, J. J.; Zakrzewski, V. G.; Dapprich, S.; Daniels, A. D.; Strain, M. C.; Farkas, O.; Malick, D. K.; Rabuck, A. D.; Raghavachari, K.; Foresman, J. B.; Ortiz, J. V.; Cui, Q.; Baboul, A. G.; Clifford, S.; Cioslowski, J.; Stefanov, B. B.; Liu, G.; Liashenko, A.; Piskorz, P.; Komaromi, I.; Martin, R. L.; Fox, D. J.; Keith, T.; Al-Laham, M. A.; Peng, C. Y.; Nanayakkara, A.; Challacombe, M.; Gill, P. M. W.; Johnson, B.; Chen, W.; Wong, M. W.; Gonzalez, C.; Pople, J. A. Gaussian 03, Revision C.02; Gaussian, Inc.: Wallingford, CT, 2004.

(39) Bussi, G.; Donadio, D.; Parrinello, M. J. Chem. Phys. 2007, 126, 014101.

(40) Parrinello, M.; Rahman, A. J. Appl. Phys. 1981, 52, 7182-7190.

(41) van der Spoel, D.; van Maaren, P. J. Chem. Theory Comput. 2006, 2, 1-11.

(42) Darden, T.; York, D.; Pedersen, L. J. Chem. Phys. 1993, 98 10089-10092.

(43) Essmann, U.; Perera, L.; Berkowitz, M. L.; Darden, T.; Lee, H.; Pedersen, L. G. J. Chem. Phys. 1995, 103, 8577-8593.

(44) Miyamoto, S.; Kollman, P. A. J. Comput. Chem. 1992, 13, $952-$ 962.

(45) Hess, B.; Bekker, H.; Berendsen, H. J. C.; Fraaije, J. G. E. M. J. Comput. Chem. 1997, 18, 1463-1472.

(46) Horovitz, A.; Matthews, J. M.; Fersht, A. R. J. Mol. Biol. 1992, $227,560-568$.

(47) Jakalian, A.; Bush, B. L.; Jack, D. B.; Bayly, C. I. J. Comput. Chem. 2000, 21, 132-146.

(48) Jakalian, A.; Jack, D. B.; Bayly, C. I. J. Comput. Chem. 2002, 23, $1623-1641$.

(49) Berendsen, H. J. C.; Postma, J. P. M.; van Gunsteren, W. F.; DiNola, A.; Haak, J. R. J. Chem. Phys. 1984, 81, 3684-3690.

(50) Cabani, S.; Gianni, P.; Mollica, V.; Lepori, L. J. Solution Chem. 1981, 10, 563-595. 
(51) Wolfenden, R.; Liang, Y. L.; Matthews, M.; Williams, R. J. Am. Chem. Soc. 1987, 109, 463-466.

(52) Abraham, M. H.; Whiting, G. S.; Fuchs, R.; Chambers, E. J. J. Chem. Soc., Perkin Trans. 2 1990, 291-300.

(53) Guthrie, J. P. Can. J. Chem. 1991, 69, 1893-1903.

(54) Guthrie, J. P.; Pike, D. C.; Lee, Y. C. Can. J. Chem. 1992, 70, $1671-1683$.

(55) Tomasi, J.; Mennucci, B.; Cammi, R. Chem. Rev. 2005, 105, 2999-3094.

(56) Talhout, R; Villa, A.; Mark, A. E.; Engberts, J. B. F. N. J. Am. Chem. Soc. 2003, 125, 10570-10579.

(57) Lawrenz, M.; Baron, R.; McCammon, J. J. Chem. Theory Comput. 2009, 5, 1106-1116.

(58) Humphrey, W.; Dalke, A.; Schulten, K. J. Mol. Graphics 1996, 14, 33-38. 